\section{Peer review (continued)}

SIR-It is time that government funding agencies provided grants in support of the peer-review system. This crucial component of the scientific process can no longer be left to the goodwill of individuals because pressure of work means that reviewing has a low priority.

In Darwin's time, peer reviewing was easy. Most studies were descriptive or involved simple experiments. Today, reviewers and editors are no longer able to verify an experiment on a desk top because experiments are costly and timeconsuming. For the same reasons, accurate and detailed communication of results by publication has become increasingly important.

At the same time, the pressures on individual scientists have caused the review process to become slower and less thorough. Prominent scientists may get 50 to 100 papers a year to review from colleagues and from journals. Some recent papers in mathematics have exceeded 100 pages and the effort required to review such a work exceeds the time available to most busy scientists.

My proposal is to provide for a rotating group of paid reviewers. Grants would be given for three months of work as a reviewer. They would be given on a lottery basis to individuals with a reasonable publication record, with the number of grants given proportional to the number of publications in each field. Individuals would be paid for their time and expenses. Each person could get the grant only once in, say, five years to avoid concentrating too much influence in a few hands. A listing of reviewers' specialities would be put into a database from which journals could pick those to whom they would send papers for review. As a limited supply of reviewers would be available because of limitations on funding, journals would be best served by sending to this group of paid reviewers only papers requiring extra attention, such as very long, very difficult or controversial papers. Most papers would still have to go to volunteers. But if the volume of papers needing review was reduced by this method, then volunteer reviewers might be able to do a more thorough job on the shorter or less controversial papers.

We have accepted the fact that many special tasks must be paid for in science. Whereas time used to be given voluntarily for editing scientific journals, most now have paid editorial assistants and even editors. Statisticians and other experts are paid for their advice and National Science

\section{Correction}

The author of the letter entitled "India's too costly imports" (Nature 340, 94; 1989) is Y.D Sharma.
Foundation grant-review panels are funded. Why do we not see reviewing as a form of consultation? Only by paying for someone's time - and thus providing recognition for their work - are we likely to increase the level of effort people are willing to put into reviews.

A final consideration concerns the purpose of the paid review. There are two types of reviewer, the gatekeeper and the mentor. The gatekeeper tries to find fault and reject a substantial number of papers, in the name of professional standards, and this can be done quite quickly. If someone is being paid, he or she can perhaps take the time to act more as a mentor. In this capacity, a reviewer would check the equations, assist the author with clarity or logic, and spend some time really thinking about the paper. Such careful review may require reading related papers cited by the authors or performing some calculations. But if a study takes several months or a year to carry out and write up, a careful review should take a day or two. A mentortype reviewer who does the job well may even end up as a coauthor on the paper, an added benefit of the position.

The question of funding for this system of grants of course arises. In fact, very little extra money would be required. Reviews are at present a 'hidden overhead', conducted in time paid for by grants given for other purposes. The costs of not having paid reviewers are very high. Papers are delayed for long periods waiting for reviews, and inadequate reviews do not help the authors to revise the paper for resubmission. Poorly written papers do not advance the field, and errors not detected before publication cause confusion and lower the reputation of science as a whole.

If 2,000 grants were awarded each year for three months of reviewing, then up to 200,000 papers could be reviewed thoroughly (assuming one to two days per review) at a cost of only $\$ 30$ million (salary plus overhead). The only cost is for direct labour, whereas normal research budgets include large amounts for expensive laboratory equipment and supplies. Spread over the huge budgets of the government funding agencies, this figure is a drop in the bucket.

Environmental Sciences Section,

CRAIG LOEHLE Savannah River Laboratory, 773-32A, Westinghouse Savannah River Co., Aiken, South Carolina 29808, USA

SiR-Although anonymous peer-reviewing has produced excellent results, the system has not been immune to insults and abuse (for example Craig Packer, Nature $340,10 ; 1989)$. Here is a simple protocol that could bring fresh hope and restore the respect the system deserves. I suggest that from now on the authors become anonymous and the reviewers come out of hiding. In this system, a journal editor would simply send a manuscript to a potential reviewer in the usual way but omitting the author's identity. The reviewer would have the option of remaining anonymous or having his or her identity revealed. Authors' identities should ideally have no bearing on the evaluation of a scientific piece of work.

\section{Department of Biochemistry \\ and Molecular Biology,} GOBINDA SARKAR

\section{Mayo Foundation,}

Rochester, Minnesota 55905, USA

SIR-It is an indictment of the scientific community if one of us could even be suspected of being remotely instrumental in the precipitous action taken by Craig Packer's colleague (Nature 340, 10; 1989). In practice, most editors maintain the anonymity of all reviewers by distributing unidentifiable comments to authors and fellow reviewers. An equally fair system would evolve if anonymous manuscripts were peer-reviewed. Editors can request that copies of submissions for this purpose should have their title on the abstract/ summary page without details of the author(s) or institution(s). The onus would then be on contributors to write their papers in a style that would make their ready indentification less easy. One would then hope that personal attacks, most of which may indeed never come to light, would be eradicated at once.

K. N. TSIQUAYE

A. J. ZUCKERMAN

Department of Clinical Sciences,

London School of Hygiene and Tropical Medicine,

Keppel Street, London WC1E 7HT, UK

\section{Names please}

SIR-It has been decided to do a volume of omissions from the Dictionary of National Biography from the beginnings to 1985 (the 1981-85 supplement will be published next spring).

The new volume gives people an opportunity to suggest names of those they have looked up in the $D N B$ and not found. This is the first time for a hundred years that such an opportunity has occurred. Previous volumes of the $D N B$ have not been as generous to scientists as they have been to people from other walks of life. I would therefore be glad to hear from any of your readers with names to suggest.

Dictionary of National Biography,

C.S. NICHOLLS

Oxford University Press,

Clarendon Building,

Bodleian Library,

Oxford OX1 3BG, UK 